\title{
Putative cancer stem cells in malignant pleural mesothelioma show resistance to cisplatin and pemetrexed
}

\author{
LOURDES CORTES-DERICKS, GIOVANNI L. CARBONI, RALPH A. SCHMID and GOLNAZ KAROUBI \\ Division of General Thoracic Surgery, University Hospital Berne, Berne, Switzerland
}

Received February 3, 2010; Accepted March 17, 2010

DOI: 10.3892/ijo_00000692

\begin{abstract}
Malignant pleural mesothelioma (MPM) is a lethal cancer of the mesothelium with high chemotherapeutic resistance via unknown mechanisms. A prevailing hypothesis states that cancer stem cells (CSCs) persist in tumors causing relapse after chemotherapy, thus, rendering these cells as critical targets responsible for tumor resistance and recurrence. We selected candidate CSC markers based on expansion under hypoxic conditions, a hallmark for the selection of chemoresistant cells; and investigated the expression of CSC markers: CD133, Bmi-1, uPAR and ABCG2 in three MPM cell lines and normal mesothelial cells by quantitative RTPCR. Furthermore, we evaluated the chemotherapeutic resistance associated with each CSC marker by determining the change in CSC marker-mRNA levels as an index of drugresistance following treatment with either cisplatin or pemetrexed. We demonstrate the expression of CSC markers: CD133, Bmi-1, uPAR and ABCG2 in both normal and MPM cell lines. Bmi- $1^{+}$, $\mathrm{uPAR}^{+}$and $\mathrm{ABCG} 2^{+}$cells show a distinct role in conferring chemoresistance to cisplatin and pemetrexed in the malignant setting. By contrast, these markers have no apparent participation in chemoresistance to drug treatments in normal mesothelial cells. Intriguingly, CD133 revealed chemoresistant properties in both normal mesothelial and malignant pleural mesothelioma cells. This study provides evidence of putative CSCs conferring drug-resistance to cisplatin and pemetrexed in MPM cell lines. Specific targeting of these drug-resistant cells, while considering the functional heterogeneity of the MPM subtypes, may contribute to more focused and effective chemotherapeutic regimens for malignant pleural mesothelioma.
\end{abstract}

\section{Introduction}

Malignant pleural mesothelioma (MPM) is a rare but highly aggressive disease with increasing incidence throughout the

Correspondence to: Professor Ralph Alexander Schmid, Division of General Thoracic Surgery, Berne CH-3010, Switzerland E-mail: ralph.schmid@insel.ch

Key words: uPAR, CD133, ABCG2, cisplatin, pemetrexed, mesothelioma, cancer stem cells world (1). This therapy-resistant neoplasm is classified into three histological subtypes: epithelial, sarcomatoid and biphasic (1). At present, there is no curative treatment for MPM, in part, attributed to the drug-resistant properties of tumor cells to chemotherapeutic agents (2). The standard therapeutic approach consists of a combination of chemotherapy, surgery and radiation; and is often associated with poor prognosis. Pemetrexed and cisplatin combination chemotherapy is the first-line treatment in inoperable disease with a median survival of 9-12.4 months (2). Cisplatin (cisdiamineplatinum dichloride) is a platinum-based chemotherapeutic drug belonging to the DNA-damaging agents, forming covalent links with DNA (2-4); whereas pemetrexed is a multi-target antifolate agent, active on three enzymes involved in synthesis of thymidine and purine nucleotides (5). Despite notable improvement using multi-modality therapy, disease recurrence and progression remain a problem, thus, it is imperative to determine the cells responsible for the resistance to chemotherapeutic regimens.

Cancer stem cells (CSCs) represent a rare population of cells with stem cell-like characteristics with the capacity to initiate and maintain neoplastic tissues $(6,7)$. Several recent reports have suggested the existence of injury-resistant, CSCs in clinical lung cancer biopsies as well as in established lung cancer cell lines (8-11). Selection of these cells has been primarily based on expression of putative CSC markers which include: CD133, ABCG2, Bmi-1, OCT4 and uPAR. CSCs are more resistant to chemotherapy than the differentiated cells of the tumor (12), through their quiescence, ability to repair DNA and adaptation to hypoxic environments (13). Chemoresistance, is in part, mediated by multi-drug resistance $\mathrm{ABC}$ transporter proteins (including breast cancer resistant protein, BCRP/ABCG2), which actively, in an ATPdependent manner extrude a large variety of drugs and drug conjugates from cells $(14,15)$. ABCG2 has been detected in solid tumors including non-small cell lung cancer (NSCLC) in which an involvement in drug resistance to topotecan, and a low response rate to platinum-based therapy have been documented (14).

B lymphoma Mo-MLV insertion region 1, Bmi-1, an oncogenic member of the polycomb repressive complexes (PRCs), has likewise been significantly associated with progression of NSCLC $(16,17)$ and recognized in small cell lung carcinoma (SCLC). Similarly, Urokinase plasminogen activator receptor, uPAR, a membrane glycosylphosphatidylinositol anchored 55-60-kDa glycoprotein, correlates with 
poor lung cancer survival and its suppression markedly reduces tumor metastasis (18-20). Rare $\mathrm{uPAR}^{+}$cells have been identified as putative CSCs in SCLC and overexpression of UPAR has been implicated in an orthotopic murine model of malignant pleural mesothelioma (19).

Recently, the transmembrane glycoprotein, CD133, has been used extensively as a marker of CSCs in cancer tissues. It has been implicated in drug-resistance to chemotherapeutic agents in lung tumor xenografts (8), and in NSCLC tissues and cell lines (9). The latter study highlights the transcription factor, OCT4, as having a pivotal role in maintaining the chemoradioresistant properties of lung cancer CD133+ cells. Embryonic stem cell marker, OCT4 (having the isoforms A and B), has been a focus in cancer stem cell research due to its recent prevalence in malignant settings $(9,21,22)$.

To date, no study has explored the existence of cancer stem cells and/or injury-resistant cells in MPM potentially responsible for the chemotherapeutic resistance and disease recurrence. In this study, we investigated the expression of candidate CSC markers: Bmi-1, uPAR, ABCG2, CD133, OCT4A and OCT4B in three malignant pleural mesothelioma cell lines; and determined the effect of single agent cisplatin and pemetrexed treatment on respective CSC mRNA levels.

\section{Materials and methods}

Cell culture. The H28, H2052 and MSTO211H mesothelioma cell lines (LGC Promochem; France) were cultured in RPMI (Invitrogen; Switzerland) media containing 10\% fetal bovine serum, FBS (PAA; Austria) and 2\% antibiotic/ antimycotic (Invitrogen) solution. The non-malignant human pleural mesothelial MeT5A cell line (LGC Promochem) was maintained in Med199 (Sigma; Germany) with $3.3 \mathrm{nM}$ epidermal growth factor (Invitrogen), $400 \mathrm{nM}$ hydrocortisone (Sigma), $870 \mathrm{nM}$ zinc-free insulin (Sigma), $387 \mathrm{ng} / 1$ selenious acid (Sigma), $50 \mathrm{ml}$ Trace elements B (1000x) (MediaTech; USA) and 10\% FBS. All cell lines were incubated at $37^{\circ} \mathrm{C}, 95 \% \mathrm{O}_{2}$ and $5 \% \mathrm{CO}_{2}$. Media were replenished every 2 days. For hypoxic culture conditions, cells were incubated for $48 \mathrm{~h}$ at $37^{\circ} \mathrm{C}, 1 \% \mathrm{O}_{2}, 95 \%$ humidity and $5 \% \mathrm{CO}_{2}$ using the Ruskinn hypoxic chamber technology (Ruskinn Tech. Ltd.; Leeds, UK).

XTT assay. Dilution series of 2-fold increments [(0-200 $\mu \mathrm{M}$; Cisplatin, CDDP, Bristol Myers Squibb, Switzerland) (0$500 \mu \mathrm{M}$; Pemetrexed, Eli Lilly, Switzerland)] were prepared for analysis using the XTT cell proliferation assay (Roche Chemicals, Switzerland). To determine the drug-dependent viability, cells $\left(10^{4}\right.$ cells/well in 96 -well plates $)$ were incubated in media with or without the addition of drugs. Following a $48 \mathrm{~h}$ incubation period, XTT cell proliferation reagent was added according to manufacturer's protocol and formazan production was measured spectrophotometrically $(450 \mathrm{~nm})$. Three independent experiments in triplicate were performed.

Drug treatment. For cisplatin treatments, cells were cultured to $80 \%$ confluency, followed by treatment with cisplatin at concentrations of $16 \mu \mathrm{M}$ (H28), $8 \mu \mathrm{M}$ (H2052), $1 \mu \mathrm{M}$ (MSTO211H) and $0.3 \mu \mathrm{M}$ (MeT5A). The specified concentrations correspond to the previously determined $\mathrm{IC}_{50}$ values for each respective cell line. Following the $48 \mathrm{~h}$ treatment at $37^{\circ} \mathrm{C}$, media was replenished with growth media in the absence of cisplatin, and cells were allowed to recover for an additional $48 \mathrm{~h}$. A similar protocol was applied for pemetrexed treatment $[100 \mu \mathrm{M}$ (H28 and H2052), $5 \mu \mathrm{M}$ (MSTO211H) and $0.5 \mu \mathrm{M}(\mathrm{MeT} 5 \mathrm{~A})]$. Three independent experiments in duplicate were performed for each drug.

Quantitative RT-PCR. Cell samples were collected in TRIzol (Invitrogen) followed by total RNA extraction using RNeasy kit (Qiagen, Switzerland) according to manufacturer's instructions. Complementary DNA, cDNA, was synthesized by reverse transcription (RT) of 5-8 $\mu \mathrm{g}$ of RNA using M-MLVRT (Promega, Switzerland) and random primers (Roche Diagnostics, Switzerland) and amplified with Taq Polymerase (Roche Diagnostics). Messenger RNA, mRNA, expression levels for house-keeping gene $(\beta 2 M$, Hs00984230_m1) and target genes (OCT4A, Hs03005111_g1; OCT4B, Hs00742896_S1; CD133, Hs01009254_m1; Bmi-1, Hs00180411_m1; uPAR, Hs00182181_m1; and ABCG2, Hs01055362_m1) were determined with TaqMan ${ }^{\circledR}$ 'Assay on Demand' probes (Applied Biosystems, Switzerland). cDNAs (50 ng) were subjected to quantitative RT-PCR in a $25 \mu 1$ reaction volume and analyzed in triplicate. The quantitative RT-PCR data represent the relative quantity of the target gene mRNA compared to the target gene mRNA/ $/ 32 M$ mRNA ratio determined from the human embryonic carcinoma cell line NTERA-2 (clone D1; European Collection of Cell Cultures, UK), used as a positive control for CSC marker expression. Gene expression was shown as relative amount of mRNA levels using the $\Delta \Delta \mathrm{Ct}$ method. All mRNA expression levels were based on gene amplifications at $<35$ cycles, which we considered as a reliable range of amplification.

Statistical analysis. To assess the statistical significance of differences observed in the mRNA expression levels between control and cisplatin-treated or pemetrexed-treated samples, a paired student's t-test was conducted. P-values $<0.05$ were defined as statistically significant.

\section{Results}

Genetic profile of MPM and normal mesothelial cell lines. The presence of potential CSCs in the MPM and control MeT5A cell lines were determined using quantitative RTPCR for the following markers: Bmi-1, uPAR, ABCG2, CD133, OCT4A and OCT4B. Fig. 1A-D depicts the mRNA expression levels of the markers in each cell line relative to expression levels found in NTERA-2. Equivalent levels of Bmi-1 expression were found in the MPM cell lines, all of which were significantly higher than that observed in MeT5A (Fig. 1E). Similarly, uPAR expression was upregulated in the MPM cells (Fig. 1E) with H2052 cells expressing the greatest levels (Fig. 1B). ABCG2 expression varied amongst the different cell lines with the H2052 cells expressing 3-6 fold higher levels of mRNA in comparison to H28 (Fig. 1A) and MSTO211H (Fig. 1C).

We detected CD133 gene expression in all three MPM cell lines, with a minute level of expression in the control MeT5A cells. Expression of CD133 was significantly higher 
A

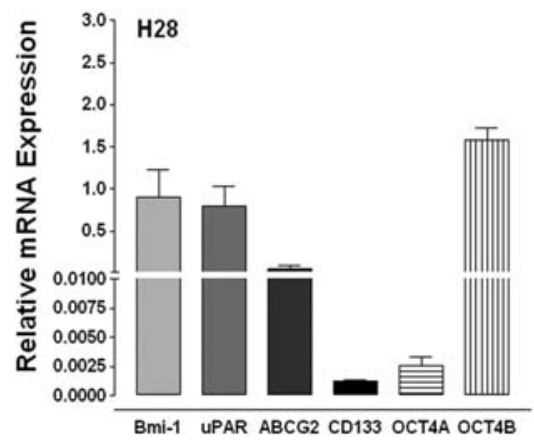

C

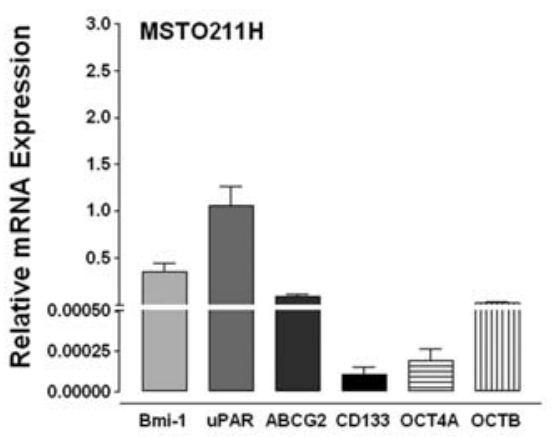

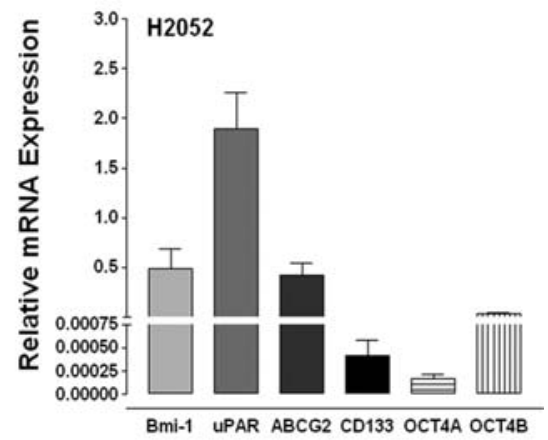

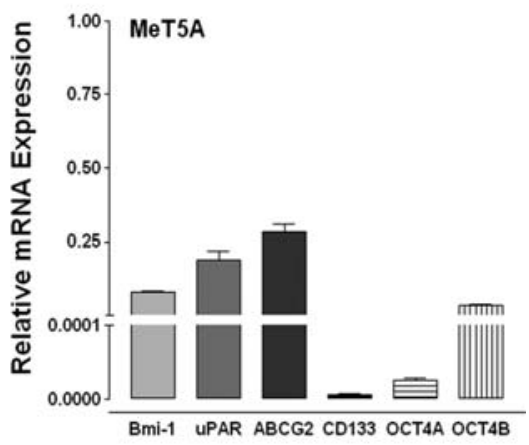

$\mathrm{E}$

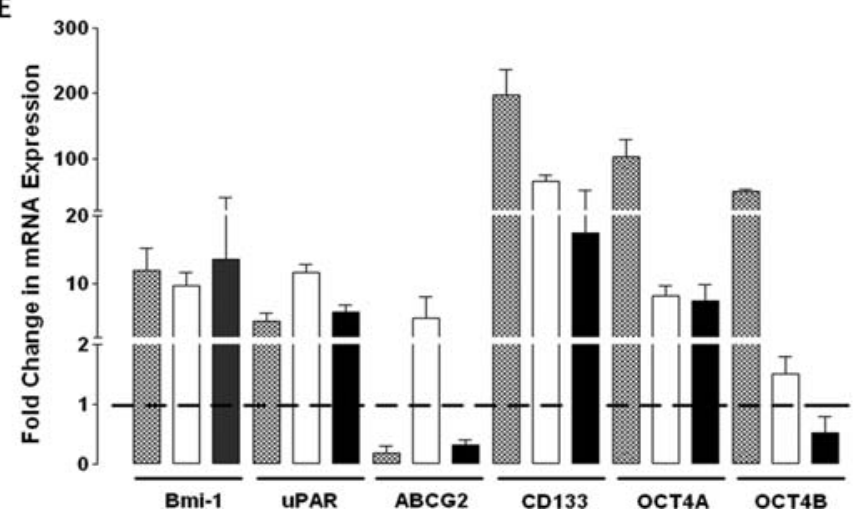

Figure 1. Basal mRNA expression of Bmi-1, uPAR, ABCG2, CD133, OCT4A and OCT4B in MPM cell lines. Quantitative RT-PCR analyses showing the basal mRNA transcript levels in (A) H28, (B) H2052, (C) MSTO211H and (D) MeT5A (control) cell lines under normoxic culture. (E) Fold change in mRNA expression levels of MPM cell lines relative to normal mesothelial MeT5A mRNA values designated at 1 (dashed line) mRNA levels were measured using the $\Delta \Delta C t$ method and relative to values obtained in the NTERA-2 embryonic carcinoma cell line.

in the H28 cell line compared to H2052 and MSTO211H, and was generally expressed at a lower level than the other markers (Fig. 1A-D). Moreover, CD133 mRNA levels were dramatically enhanced in the MPM cell lines (Fig. 1E). In the case of OCT4, established primer/probes designed to specifically target the $\mathrm{A}$ and $\mathrm{B}$ isoforms, and free from interfering pseudogenes were used (23). OCT4A mRNA levels were highest in $\mathrm{H} 28$ cells, and low but detectable in the H2052, MSTO211H and MeT5A cells. In contrast, significantly higher levels of OCT4B (as compared to OCT4A) were observed in all cell lines including MeT5A with no significant rise in expression levels in the MPM cell lines.

Effect of hypoxia on the expression of CSC markers. Hypoxia (low oxygen tension) is a selective insult for genomic alterations that lead to chemotherapeutic resistance observed in
CSC populations (13). Therefore, we selected our candidate CSCs in MPM cell lines (H28, H2052 and MSTO211H) based on CSC expansion under hypoxic culture. Cells were placed under a hypoxic environment for $48 \mathrm{~h}$ after which we assessed for changes in CSC mRNA expression levels compared to normal growth under normoxic settings (Fig. 2). As expected, hypoxia induced elevated levels of ABCG2 (24) and CD133 (25). In addition, we detected a significant increase in UPAR expression levels. This is in agreement with recent studies demonstrating enhanced UPAR expression in tumor-derived cells cultured in hypoxic conditions (26). Interestingly, hypoxia had a considerable effect on Bmi-1 expression levels only in the MSTO211H cell line. In contrast to several reports, there was no increase in either OCT4A or OCT4B under hypoxic conditions in any of the MPM cell lines. It is important to note, however, that it is plausible for 
A

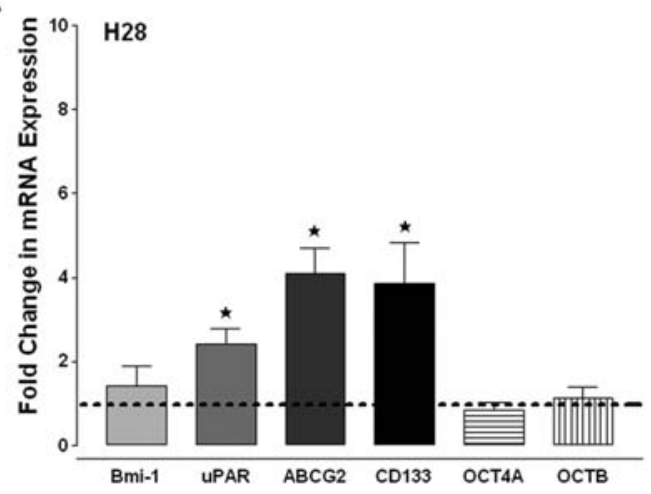

B

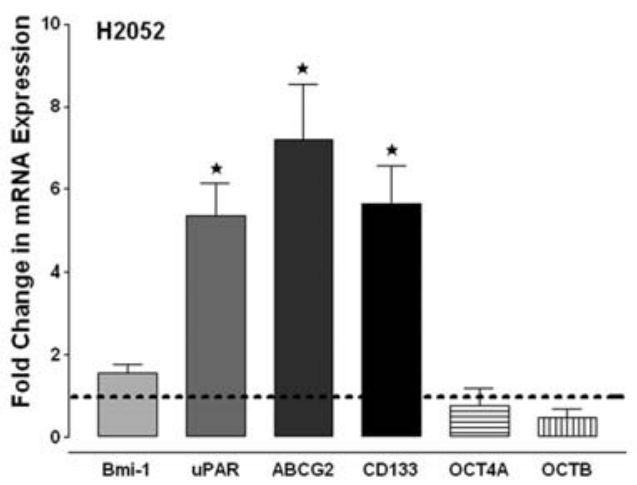

C

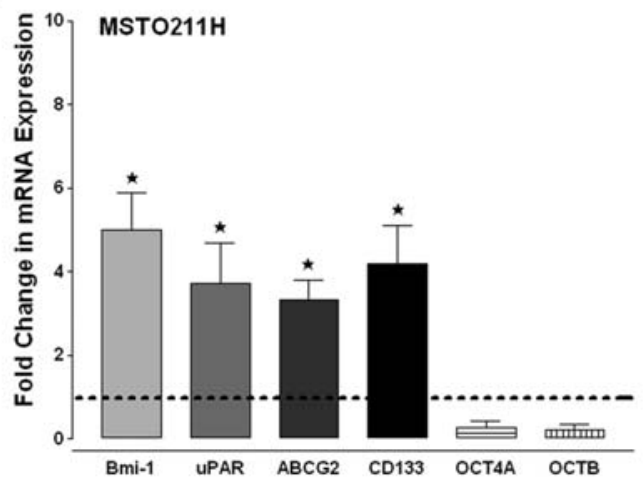

Figure 2. Expression of Bmi-1, uPAR, ABCG2, CD133, OCT4A and OCT4B under hypoxic environment. Quantitative RT-PCR analyses showing the fold changes of mRNA transcripts in $\mathrm{H} 28, \mathrm{H} 2052$ and MSTO211H under hypoxic setting compared to the normoxic basal expression with an assigned value of 1 (dashed line).

OCT4 to have an oncogenic role in MPM and function as a regulator of chemoradioresistance in cancer stem cell populations (9). Nevertheless, due to the lack of increase of OCT4 expression in MPM cell lines in hypoxic culture, OCT4A and OCT4B were not evaluated in the subsequent drug treatment experiments.

Effect of cisplatin and pemetrexed treatments on cell viability. To determine the effect of the different concentrations of cisplatin and pemetrexed on cell viability, cells were treated with increasing concentrations of either cisplatin or pemetrexed (Fig. 3). MPM cell lines portrayed relatively variable cell sensitivity levels to cisplatin with an $\mathrm{IC}_{50}$ of 16,8 and $1 \mu \mathrm{M}$ for $\mathrm{H} 28, \mathrm{H} 2052$ and MSTO211H cells, respectively. Higher resistance to pemetrexed was recognized in all MPM
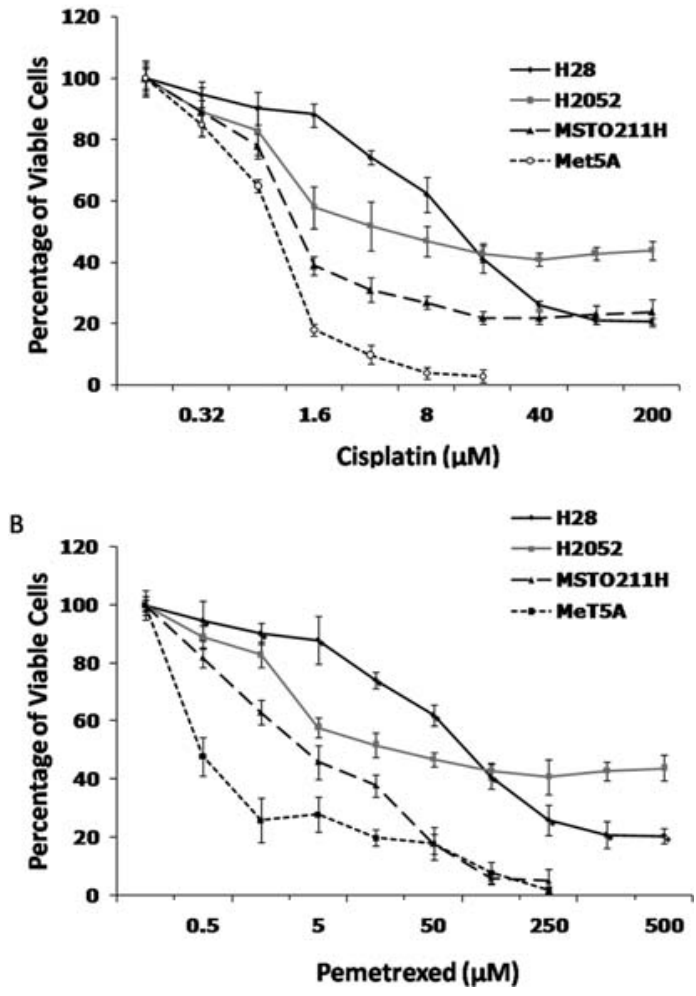

Figure 3. Dose-response curves of MPM cell lines in the presence of either cisplatin or pemetrexed. Effects of different concentrations of (A) cisplatin $(0-200 \mu \mathrm{M})$ and $(\mathrm{B})$ pemetrexed $(0-500 \mu \mathrm{M})$ on the cell viability of $\mathrm{H} 28$, $\mathrm{H} 2052$, MSTO211H and MeT5A as determined by the XTT cell proliferation assay. Results represent the means \pm SD of three independent experiments.

cell lines cells with an $\mathrm{IC}_{50}$ of $100 \mu \mathrm{M}$ for both $\mathrm{H} 2052$ and $\mathrm{H} 28$ cells. Note that a much lower $\mathrm{IC}_{50}(5 \mu \mathrm{M})$ was observed for the MSTO211H cell line. MeT5A cells had IC $_{50}$ values of $0.3 \mu \mathrm{M}$ for cisplatin and $0.5 \mu \mathrm{M}$ for pemetrexed, respectively.

Effect of cisplatin treatment on CSC expression levels. Cisplatin treatment of $\mathrm{H} 28$ cells resulted in a significant increase in UPAR $(p=0.002)$ and ABCG2 $(p=0.02)$ compared to the non-treated paired controls. In addition, there was a slight increase in Bmi-1 expression although this value did not reach statistical significance. In contrast, there was a significant reduction in CD133 $(p=0.04)$ after cisplatin treatment suggesting that $\mathrm{CD} 133^{+}$cells in $\mathrm{H} 28$ are sensitive to cisplatin (Fig. 4A). Taken together, these results indicate that in the H28 cell line, ABCG2 and UPAR may be in part, responsible for the resistance to platinum-based chemotherapeutic drugs.

Despite a slight increase in UPAR expression, treatment of H2052 cells with cisplatin did not show any marked increase in the expression of CSC markers (Fig. 4B). In MSTO211H cells, cisplatin treatment showed an upregulation of Bmi-1 $(p=0.04), \operatorname{ABCG} 2(p=0.03)$ and CD133 $(p=0.001)$ (Fig. 4C). Treatment of MeT5A cells resulted in decreased levels of the majority of CSC markers, significantly reducing uPAR $(p=0.0001)$ and ABCG2 $(p=0.008)$ mRNA levels (Fig. 4D). Interestingly, only CD133 was dramatically increased in the control MeT5A cells suggestive of an innate 
A

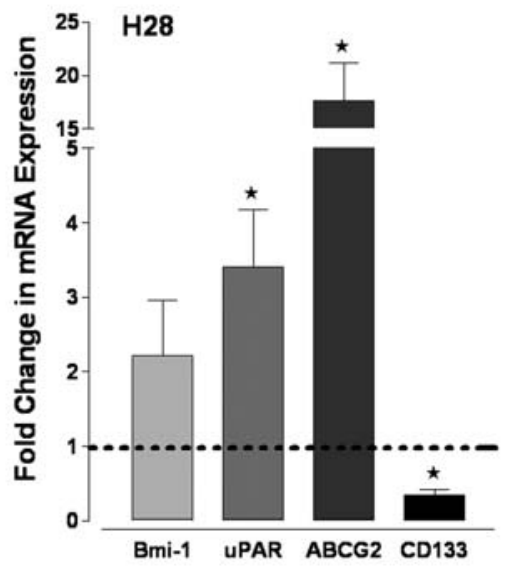

C

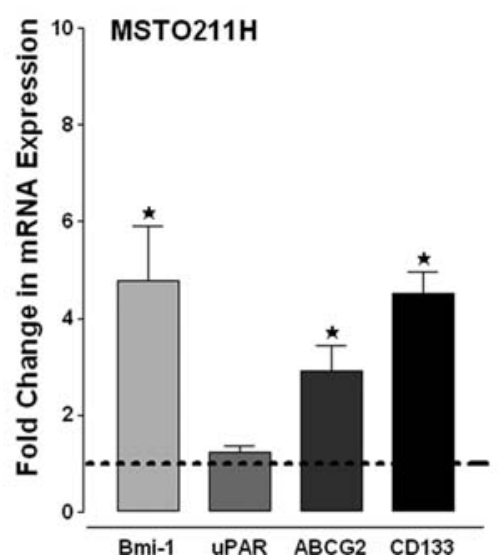

B

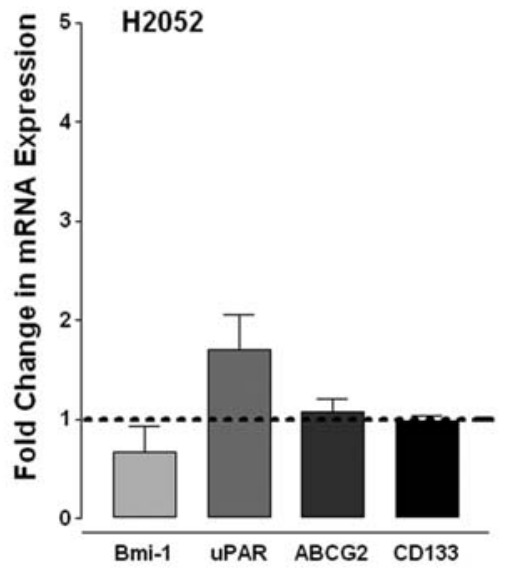

D

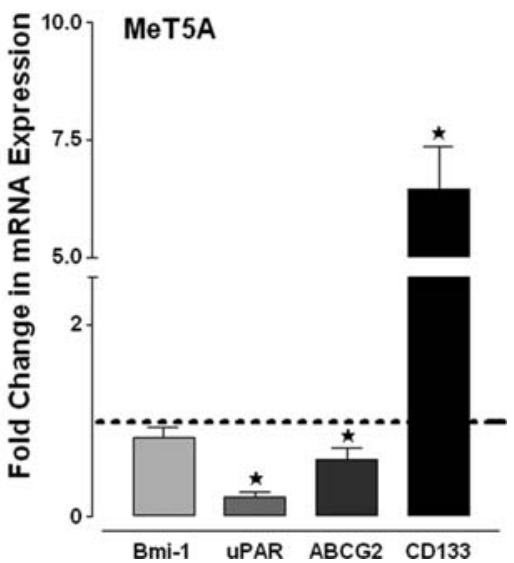

Figure 4. Effect of cisplatin on the expression of candidate CSC markers. Fold changes in the relative mRNA expression of Bmi-1, uPAR, ABCG2 and CD133 following treatments in the absence or presence of cisplatin in (A) H28, (B) H2052, (C) MSTO211H and (D) MeT5A. Non-treated cells were taken as controls with assigned values of 1 (dashed lines). Results represent the means \pm SD of a minimum of three independent experiments.

injury-resistive property of $\mathrm{CD} 133^{+}$cells in a non-malignant setting.

Effect of pemetrexed treatment on CSC expression levels. Treatment of $\mathrm{H} 28$ cells with pemetrexed resulted in significant elevation of uPAR $(p=0.001)$ and ABCG2 $(p=0.0004)$ mRNA levels. However, no changes in Bmi-1 and CD133 were observed following pemetrexed treatment (Fig. 5A). Notably, there was a marked increase in CD133 $(p=0.02)$ in the H2052 cells indicative of a potential role for CD133+ cells in pemetrexed resistance in this cell line (Fig. 5B). Similar to the effects of cisplatin treatments, results with pemetrexed did not show considerable change in Bmi-1 expression levels in either $\mathrm{H} 28$ or $\mathrm{H} 2052$ cells. Pemetrexed treatment of MSTO211H cells revealed a significant upregulation of all putative CSC markers (Fig. 5C). Further, pemetrexed treatment of MeT5A cells reduced Bmi-1 $(\mathrm{p}=0.004)$ and ABCG2 ( $\mathrm{p}=0.0002)$ expression and significantly raised CD133 mRNA levels ( $\mathrm{p}=0.00003)$ (Fig. 5D).

\section{Discussion}

In this study, we demonstrate for the first time expression of CSC markers: CD133, Bmi-1, uPAR and ABCG2 in three malignant pleural mesothelioma cell lines H28, H2052 and MSTO211H. We also show that these expression levels are significantly higher than those observed in the non-malignant pleural mesothelial cells, MeT5A. Our data reveal that in normal mesothelial cells, Bmi-1, uPAR and ABCG2 have no apparent participation in chemoresistance to single agent cisplatin and pemetrexed treatments. By contrast, chemotherapeutic treatments convey a distinct role for $\mathrm{Bmi}-1^{+}$, $\mathrm{uPAR}^{+}$and $\mathrm{ABCG}^{+}$cells in conferring chemoresistance to these drugs in the malignant setting. This conforms to the cancer stem cell paradigm indicating the direct participation of CSCs in chemoresistance (27). Intriguingly, CD133 revealed chemoresistant properties in both normal mesothelial cells and malignant pleural mesothelioma cells. In summary, our results suggest the existence of putative drugresistant CSCs in the three MPM cell lines. Importantly, the degree of drug-resistance elicited by CSCs depends on the specific chemotherapeutic agent used, and the subtype of MPM investigated.

CSCs are hypothesized to be the origin of replicating malignant tumor cells and have been isolated from a variety of solid tumors. The presence of CSCs in tumors may result in enhanced resistance to conventional chemotherapeutic regimens, thus, impairing long-term survival after therapy 
A

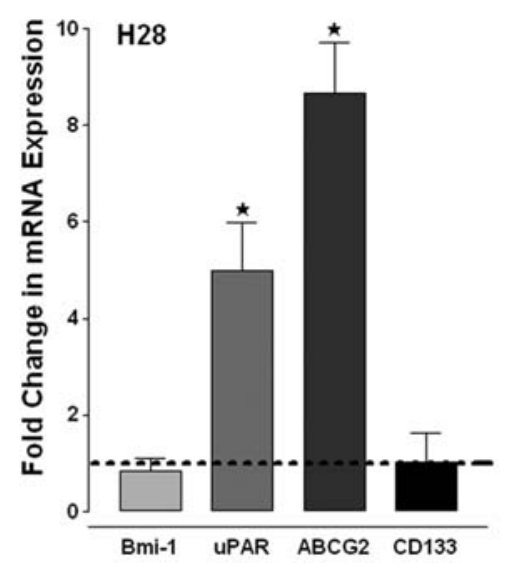

C

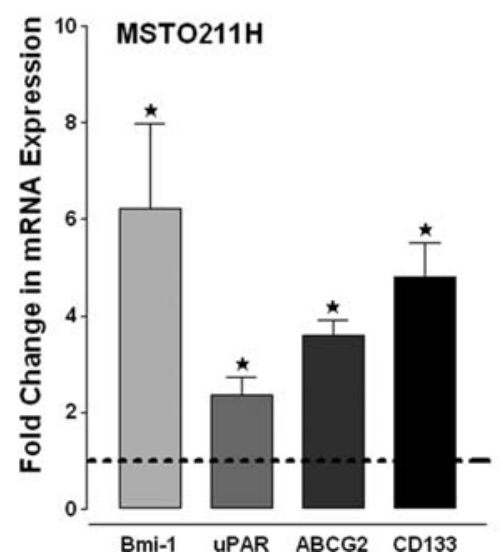

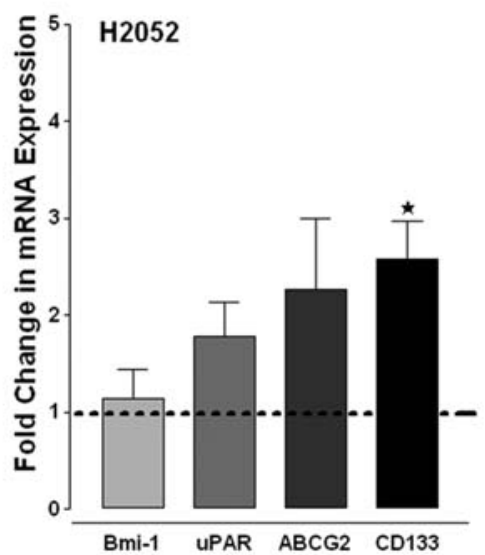

D

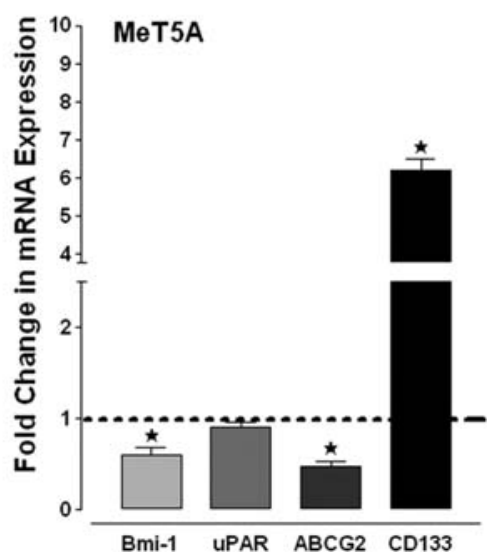

Figure 5. Effect of pemetrexed on the expression of candidate CSC markers. Fold changes in the relative mRNA expression of Bmi-1, uPAR, ABCG2 and CD133 following treatments in the absence or presence of pemetrexed in (A) H28, (B) H2052, (C) MSTO211H and (D) MeT5A. Non-treated cells were taken as controls with assigned values of 1 (dashed lines). Results represent the means \pm SD of a minimum of three independent experiments.

(6). In the lung, putative CSC markers include: CD133 $(8,10)$, embryonic stem cell marker OCT4 (9), multi-drug resistant protein ABCG2 $(28)$, Bmi-1 $(16,29)$ and uPAR $(20,30)$.

Treatment with cisplatin and pemetrexed illustrated a significant increase in $\mathrm{ABCG} 2$ expression in $\mathrm{H} 28$ and MSTO211H cells inferring a potential role for ABCG2 in the resistance to these chemotherapeutic drugs. Overexpression of $\mathrm{ABC}$ transporters reduces the formation of platinum-DNA adducts in tumor cells, resulting in resistance to platinumbased drugs such as cisplatin (31). A study of 156 NSCLC tumors showed that negativity for ABCG2 expression was a positive prognostic factor for advanced NSCLC (32). Likewise, a much lower response rate has been reported following platinum-based chemotherapy in patients with ABCG2 positive tumors (14).

There was no significant ABCG2-mediated drugresistance following cisplatin and pemetrexed treatments in the H2052 cells. This response to pemetrexed treatment could be attributed to complex pharmacological mechanisms between multi-drug resistant $\mathrm{ABC}$ transporters and antifolate properties of the drug. The threshold of $\mathrm{ABC}$ transporter expression in pathological conditions is critical for antifolate resistance. High expression of ABCG2, results in increased folate efflux, thereby leading to reduction of the intracellular folate pool, and a subsequent overall decrease in antifolate resistance (5). The high basal expression of ABCG2 in the H2052 MPM cell could result in reduced intracellular folate pools, and hence, decreased ABCG2-mediated antifolate resistance.

CD133 was first recognized in $\mathrm{CD}_{3} 4^{+}$progenitor populations from adult blood and bone marrow and high levels of CD133 expression is now accepted as a characteristic for hematopoietic and neural stem cells (33-35). We detected low levels of CD133 in the MeT5A non-malignant human pleural cells. Further, we found that $\mathrm{CD} 133^{+}$cells are resistant to treatment with chemotherapeutic drugs further supporting CD133 as a potential marker for normal mesothelial progenitor cells with innate injury resistive properties. CD133 has been shown to be highly expressed in CSC populations $(6,36-$ 39), and CD133+ CSCs have been deemed to be resistant to chemotherapy treatment $(8,10,40)$. The most recent evidence for CD133 as a CSC marker in the lung was presented by Bertolini et al (8) who illustrate a cisplatin-resistant CD133+ subpopulation in primary NSCLC. In agreement with these reports, we observed an upregulation of CD133 in the H2052 and MSTO211H cells following treatment with pemetrexed and in the case of MSTO211H, cisplatin treatment. However, 
recent reports have also questioned the validity of CD133 as a marker for cancer stem cells and its functional relevance for tumor-initiation (41). In agreement with these reports, we found that treatments with cisplatin or pemetrexed did not result in elevated CD133 expression levels in H28 cells, and instead, showed a marked CD133 reduction after cisplatin treatment which indicates sensitivity to such treatment.

uPAR is broadly distributed in MPM cells and results in induction of cell response to various stimuli implicated in the pathogenesis of MPM (30). Expression of uPAR has also been found in harvested tissue from MPM patients (30). Here we observe a 4-8 fold increase in basal uPAR mRNA expression levels in the MPM cell lines in comparison to MeT5A cells. This is in agreement with Tucker et al (19) who demonstrate similar levels of increased UPAR protein expression in their tested MPM cell lines. We found that treatment with cisplatin and pemetrexed resulted in a marked increase in uPAR expression in $\mathrm{H} 28$ and MSTO211H suggestive of a chemoresistive function of $\mathrm{uPAR}^{+}$cells in these cell lines. It has been shown that UPAR expression promotes cell survival by activating anti-apoptotic factor Bcl-XL transcription, through the MEK/ERK and PI3K/Akt dependent pathways. Whether the anti-apoptotic mechanism, resulting from increased UPAR expression, confers chemoresistance to the MPM cell lines remains to be investigated.

Bmi-1 has been identified as a member of a death-fromcancer signature profile in a multitude of human cancers and specifically as an oncoprotein in NSCLC (16). Despite its likely oncogenic role in NSCLC, to date, no study has shown Bmi-1 expression in malignant pleural mesothelioma. We found an approximately 5-12 fold increase in basal Bmi-1 mRNA expression levels in the MPM cell lines compared to that in MeT5A. Furthermore, Bmi-1 expression was significantly increased following cisplatin and pemetrexed treatment in MSTO211H cells. Although it has been suggested that elevated expression of Bmi-1 is an early event in the development of lung cancer (16), the exact mechanism of Bmi-1 upregulation still remains to be elucidated. Unlike the MSTO211H cell line, there appears to be no function for Bmi-1 in conferring chemoresistance to cisplatin and pemetrexed in the H28 and H2052 MPM cell lines.

Several recent studies have elucidated functional variances and different responses to chemotherapy between MPM subtypes $(42,43)$. Our results indicate that there are notable differences between epithelial, sarcomatoid and biphasic MPM cell lines with respect to the expression levels of candidate CSC markers, and their response to cisplatin and pemetrexed treatments. It is, therefore, important to take into consideration the cellular and functional heterogeneity of MPM in designing therapeutic regimens for a more effective treatment. This is an important first study, illustrating increased expression levels of putative CSCs with chemoresistive properties in malignant pleural mesothelioma cell lines and warrants further investigation exploring CSC-mediated drugresistance.

\section{Acknowledgements}

Funding for this project was from grant no. 103 provided by the Bernese Cancer League in Berne, Switzerland.

\section{References}

1. Pistolesi M and Rusthoven J: Malignant pleural mesothelioma: update, current management, and newer therapeutic strategies. Chest 126: 1318-1329, 2004.

2. Vandermeers F, Hubert P, Delvenne P, et al: Valproate, in combination with pemetrexed and cisplatin, provides additional efficacy to the treatment of malignant mesothelioma. Clin Cancer Res 15: 2818-2828, 2009.

3. Maziak DE, Gagliardi A, Haynes AE, Mackay JA and Evans WK: Surgical management of malignant pleural mesothelioma: a systematic review and evidence summary. Lung Cancer 48: 157-169, 2005.

4. Zellos L and Christiani DC: Epidemiology, biologic behavior, and natural history of mesothelioma. Thorac Surg Clin 14: 469-477, viii, 2004.

5. Assaraf YG: Molecular basis of antifolate resistance. Cancer Metastasis Rev 26: 153-181, 2007.

6. O' Brien CA, Kreso A and Dick JE: Cancer stem cells in solid tumors: an overview. Semin Radiat Oncol 19: 71-77, 2009.

7. Gilbert CA and Ross AH: Cancer stem cells: cell culture, markers, and targets for new therapies. J Cell Biochem (In press.

8. Bertolini G, Roz L, Perego P, et al: Highly tumorigenic lung cancer $\mathrm{CD}_{133^{+}}$cells display stem-like features and are spared by cisplatin treatment. Proc Natl Acad Sci USA 106: 16281-16286, 2009.

9. Chen YC, Hsu HS, Chen YW, et al: Oct-4 expression maintained cancer stem-like properties in lung cancer-derived CD133-positive cells. PLoS One 3: e2637, 2008.

10. Tirino V, Camerlingo R, Franco R, et al: The role of CD133 in the identification and characterisation of tumour-initiating cells in non-small-cell lung cancer. Eur J Cardiothorac Surg 36: 446-453, 2009.

11. Eramo A, Lotti F, Sette G, et al: Identification and expansion of the tumorigenic lung cancer stem cell population. Cell Death Differ 15: 504-514, 2008.

12. Bao S, Wu Q, Sathornsumetee $\mathrm{S}$, et al: Stem cell-like glioma cells promote tumor angiogenesis through vascular endothelial growth factor. Cancer Res 66: 7843-7848, 2006.

13. Silvan U, Diez-Torre A, Arluzea J, Andrade R, Silio M and Arechaga J: Hypoxia and pluripotency in embryonic and embryonal carcinoma stem cell biology. Differentiation 78: 159-168, 2009.

14. Yoh K, Ishii G, Yokose T, et al: Breast cancer resistance protein impacts clinical outcome in platinum-based chemotherapy for advanced non-small cell lung cancer. Clin Cancer Res 10: 1691-1697, 2004

15. Harada T, Ogura S, Yamazaki K, et al: Predictive value of expression of P53, Bcl-2 and lung resistance-related protein for response to chemotherapy in non-small cell lung cancers. Cancer Sci 94: 394-399, 2003.

16. Glinsky GV, Berezovska O and Glinskii AB: Microarray analysis identifies a death-from-cancer signature predicting therapy failure in patients with multiple types of cancer. J Clin Invest 115: 1503-1521, 2005.

17. Vrzalikova K, Skarda J, Ehrmann J, et al: Prognostic value of Bmi-1 oncoprotein expression in NSCLC patients: a tissue microarray study. J Cancer Res Clin Oncol 134: 1037-1042, 2008.

18. Alfano D, Iaccarino I and Stoppelli MP: Urokinase signaling through its receptor protects against anoikis by increasing BCL-XL expression levels. J Biol Chem 281: 17758-17767, 2006.

19. Tucker TA, Dean C, Komissarov A, et al: The urokinase receptor supports tumorigenesis of human malignant pleural mesothelioma cells. Am J Respir Cell Mol Biol (In press).

20. Gutova M, Najbauer J, Gevorgyan A, et al: Identification of uPAR-positive chemoresistant cells in small cell lung cancer. PLoS One 2: e243, 2007.

21. Sotomayor P, Godoy A, Smith GJ and Huss WJ: Oct4A is expressed by a subpopulation of prostate neuroendocrine cells. Prostate 69: 401-410, 2009.

22. Monsef N, Soller M, Isaksson M, Abrahamsson PA and Panagopoulos I: The expression of pluripotency marker Oct 3/4 in prostate cancer and benign prostate hyperplasia. Prostate 69: 909-916, 2009.

23. Cantz T, Key G, Bleidissel M, et al: Absence of OCT4 expression in somatic tumor cell lines. Stem Cells 26: 692-697, 2008. 
24. Comerford KM, Wallace TJ, Karhausen J, Louis NA, Montalto MC and Colgan SP: Hypoxia-inducible factor-1-dependent regulation of the multidrug resistance (MDR1) gene. Cancer Res 62: 3387-3394, 2002.

25. Platet N, Liu SY, Atifi ME, et al: Influence of oxygen tension on CD133 phenotype in human glioma cell cultures. Cancer Lett 258: 286-290, 2007.

26. Chaudary N and Hill RP: Increased expression of metastasisrelated genes in hypoxic cells sorted from cervical and lymph nodal xenograft tumors. Lab Invest 89: 587-596, 2009.

27. Dean M, Fojo T and Bates S: Tumour stem cells and drug resistance. Nat Rev Cancer 5: 275-284, 2005.

28. Smith PJ, Furon E, Wiltshire M, et al: ABCG2-associated resistance to Hoechst 33342 and topotecan in a murine cell model with constitutive expression of side population characteristics. Cytometry A 75: 924-933, 2009.

29. Koch LK, Zhou H, Ellinger J, et al: Stem cell marker expression in small cell lung carcinoma and developing lung tissue. Hum Pathol 39: 1597-1605, 2008.

30. Idell S, Pueblitz S, Emri S, et al: Regulation of fibrin deposition by malignant mesothelioma. Am J Pathol 147: 1318-1329, 1995.

31. Cervenak J, Andrikovics H, Ozvegy-Laczka C, et al: The role of the human ABCG2 multidrug transporter and its variants in cancer therapy and toxicology. Cancer Lett 234: 62-72, 2006.

32. Ota S, Ishii G, Goto K, et al: Immunohistochemical expression of BCRP and ERCC1 in biopsy specimen predicts survival in advanced non-small-cell lung cancer treated with cisplatinbased chemotherapy. Lung Cancer 64: 98-104, 2009.

33. Barraud P, Stott S, Mollgard K, Parmar M and Bjorklund A: In vitro characterization of a human neural progenitor cell coexpressing SSEA4 and CD133. J Neurosci Res 85: 250-259, 2007.

34. Hicks C, Wong R, Manoharan A and Kwan YL: Viable $\mathrm{CD} 34^{+} / \mathrm{CD} 133^{+}$blood progenitor cell dose as a predictor of haematopoietic engraftment in multiple myeloma patients undergoing autologous peripheral blood stem cell transplantation. Ann Hematol 86: 591-598, 2007.
35. He X, Gonzalez V, Tsang A, Thompson J, Tsang TC and Harris DT: Differential gene expression profiling of CD34 $4^{+}$ $\mathrm{CD}_{133^{+}}$umbilical cord blood hematopoietic stem progenitor cells. Stem Cells Dev 14: 188-198, 2005.

36. Piccirillo SG and Vescovi AL: Bone morphogenetic proteins regulate tumorigenicity in human glioblastoma stem cells. Ernst Schering Found Symp Proc, pp59-81, 2006.

37. Singh S and Dirks PB: Brain tumor stem cells: identification and concepts. Neurosurg Clin N Am 18: 31-38, viii, 2007.

38. Richardson GD, Robson CN, Lang SH, Neal DE, Maitland NJ and Collins AT: CD133, a novel marker for human prostatic epithelial stem cells. J Cell Sci 117: 3539-3545, 2004.

39. Ricci-Vitiani L, Lombardi DG, Pilozzi E, et al: Identification and expansion of human colon-cancer-initiating cells. Nature 445: 111-115, 2007.

40. Chang CJ, Hsu CC, Yung MC, et al: Enhanced radiosensitivity and radiation-induced apoptosis in glioma CD133-positive cells by knockdown of SirT1 expression. Biochem Biophys Res Commun 380: 236-242, 2009.

41. Horst D, Scheel SK, Liebmann S, et al: The cancer stem cell marker CD133 has high prognostic impact but unknown functional relevance for the metastasis of human colon cancer. J Pathol (In press).

42. Sun X, Wei L, Liden J, et al: Molecular characterization of tumour heterogeneity and malignant mesothelioma cell differentiation by gene profiling. J Pathol 207: 91-101, 2005.

43. Sorensen JB, Frank H and Palshof T: Cisplatin and vinorelbine first-line chemotherapy in non-resectable malignant pleural mesothelioma. Br J Cancer 99: 44-50, 2008. 